\title{
A Polarimetric Radar Approach to Identify Rain, Melting-Layer, and Snow Regions for Applying Corrections to Vertical Profiles of Reflectivity
}

\author{
SERGEY Y. Matrosov \\ Cooperative Institute for Research in Environmental Sciences, University of Colorado, and NOAA/Earth System Research \\ Laboratory, Boulder, Colorado \\ Kurt A. Clark \\ NOAA/Earth System Research Laboratory, Boulder, Colorado \\ DAVID E. KINGSMILL \\ Cooperative Institute for Research in Environmental Sciences, University of Colorado, and NOAA/Earth System Research \\ Laboratory, Boulder, Colorado
}

(Manuscript received 5 September 2006, in final form 7 November 2006)

\begin{abstract}
This article describes polarimetric X-band radar-based quantitative precipitation estimations (QPE) under conditions of low freezing levels when, even at the lowest possible elevation angles, radar resolution volumes at longer ranges are in melting-layer or snow regions while it rains at the ground. A specifically adjusted vertical-profile-of-reflectivity (VPR) approach is introduced. The mean VPR is constructed based on the range-height indicator scans, and the effects of smoothing of brightband (BB) features with range are accounted for. A principal feature of the suggested QPE approach is the determination of the reflectivity $\mathrm{BB}$ boundaries and freezing-level heights on a beam-by-beam basis using the copolar correlation coefficient $\rho_{\mathrm{hv}}$, which is routinely available from the X-band radar measurements. It is shown that this coefficient provides a robust discrimination among the regions of rain, melting hydrometeors, and snow. The freezing-level estimates made using $\rho_{\text {hy }}$ were within 100-200 $\mathrm{m}$ from the corresponding estimates of the $0^{\circ}$ isotherm heights from radiosonde soundings. The suggested VPR approach with the polarimetric determination of the reflectivity BB boundaries was used for QPE during the wintertime deployment of the NOAA X-band radar as part of the 2006 Hydrometeorological Test Bed (HMT-06) field experiment in the California Sierra Nevada foothills. It is shown that this approach noticeably improves radar-rainfall accumulation estimates. The use of the HMT-06 mean X-band reflectivity-rain-rate $\left(Z_{\mathrm{eh}}-R\right)$ relation resulted in an approximately $65 \%$ relative standard deviation of radar estimates from the surface rain gauges if no VPR adjustments were made. Applying the VPR approach with polarimetric detection of the melting layer resulted in reduction of the corresponding relative standard deviation by about a factor of 2 .
\end{abstract}

\section{Introduction}

A recent field experience gained with the use of the National Oceanic and Atmospheric Administration (NOAA) transportable polarimetric X-band $(3.2-\mathrm{cm}$ wavelength, $\lambda$ ) radar (e.g., Matrosov et al. 2002, 2005) and with other radars operating at similar frequencies (e.g., Anagnostou et al. 2004; Park et al. 2005) showed

Corresponding author address: Dr. Sergey Matrosov, R/PSD2, 325 Broadway, Boulder, CO 80305.

E-mail: sergey.matrosov@noaa.gov

DOI: $10.1175 / J A M 2508.1$

(C) 2007 American Meteorological Society that X-band systems $(\lambda \sim 3 \mathrm{~cm})$ are capable of providing high-resolution and reasonably accurate estimates of rainfall parameters within a range of several dozens of kilometers, given that radar echoes do not have significant contributions from mixed-phase and snow regions of precipitating systems. The progress in X-band radar-rainfall measurements is, to a significant extent, due to an introduction of polarimetric approaches to account for attenuation and differential attenuation effects, which used to be a major obstacle for a wider use of X-band frequencies for quantitative precipitation estimates (QPE). These approaches are based on differential-phase-shift measurements between horizontally 
and vertically polarized radar signals and provide a relatively robust way to correct measurements of reflectivity $Z_{\mathrm{eh}}$ and differential reflectivity $Z_{\mathrm{DR}}$.

Given that the attenuation and differential attenuation effects are taken care of (or, at least, mitigated to an acceptable degree of correction uncertainties), important logistical advantages of X-band radars, such as their relatively small size and cost, make them an attractive choice for a number of practical applications. These applications include the use of X-band radars to fill gaps in the operational weather service radar network coverage and to provide high-resolution rainfall measurements over important watersheds and basins. At X-band frequencies, propagation differential-phaseshift signals are stronger in comparison with C-band $(\lambda \sim 5 \mathrm{~cm})$ and S-band $(\lambda \sim 10 \mathrm{~cm})$ frequencies, which are traditionally used with weather radars. This fact makes it possible to apply some polarimetric QPE rainfall approaches for rain rates $R$ as low as $2-3 \mathrm{~mm} \mathrm{~h}^{-1}$, whereas an effective use of such approaches at longer wavelengths becomes possible only at larger values of $R$ (Matrosov et al. 2006).

As part of the Hydrometeorological Test Bed (HMT06) project, the NOAA X-band radar was deployed during the winter of 2005/06 in the American River basin in the California Sierra Nevada foothills near the town of Auburn at an elevation of $460 \mathrm{~m}$ above mean sea level (MSL). In a QPE mode, the radar scanned in the northeast quadrant with a maximum range of 38 $\mathrm{km}$. Because of the gradually rising terrain in the direction of scanning, $3^{\circ}$ was the lowest possible radar elevation angle that provided an unobstructed horizon in the $0^{\circ}-90^{\circ}$ sector of interest. Most of the storms observed during the HMT-06 field project had a freezing level (i.e., the height of the $0^{\circ} \mathrm{C}$ isotherm) located between approximately 0.5 and $2 \mathrm{~km}$ above the ground level (AGL). An average lower boundary of the reflectivity bright band of about $1 \mathrm{~km}$ AGL and the lowest possible radar elevation angle of $3^{\circ}$ resulted in a muchreduced radius $(\sim 17-19 \mathrm{~km}$, on average) of a sector area within which the radar actually was sensing rain and polarimetric QPE for rainfall could be used.

At longer ranges, even when it was raining at the ground, the radar resolution volume was partially or completely filled with either the mixed-phase hydrometeors in a melting layer or with snow particles above the freezing level. Such situations often happen with observations with longer-wavelength operational radars when, even at the lowest elevation angles $\left(\sim 0.5^{\circ}\right)$, radar beams broaden and increase in height with range. One of the approaches suggested to account for these effects when applying reflectivity-based rainfall estimators is to use a priori information on the vertical profile of reflectivity (VPR; e.g., Andrieu and Creutin 1995; Bellon et al. 2005). Under this approach the reflectivity measured aloft is related in a mean sense to an expected reflectivity at the ground. This ground-level reflectivity estimate is then used for QPE. It was shown (e.g., Koistinen 1991) that for ranges of less than 100 $\mathrm{km}$ a VPR correction can improve reflectivity-based rainfall estimates by a factor that is between 1.5 and 2 .

In this study, a modified VPR approach for rainfall estimates is suggested. It is specifically tailored to Xband polarimetric radar measurements, but the general concept also applies to polarimetric radars at other wavelengths. The main feature of this approach is that the polarimetric data are used to identify the location and the extent of the melting layer for each particular beam of radar data rather than utilizing an average a priori VPR and melting-layer heights based on climatological or other auxiliary data. Because the polarimetric QPE in mixed phase and snow remains a largely unexplored area, X-band polarimetric capabilities are used here mainly for determining melting layers and to correct reflectivities for attenuation at shorter ranges filled with rain. Mean reflectivity-based estimators are used for QPE once reflectivity values at the ground are estimated based on the mean VPR and beam-specific information on locations of melting layers.

\section{X-band vertical profiles of reflectivity during HMT-06}

Between the routine low-elevation-angle scans in the QPE mode, the NOAA X-band radar performed regular range-height indicator (RHI) scans within a sector of interest (i.e., $0^{\circ}-90^{\circ}$ in azimuth). These RHI scans were used to construct the VPR. Two representative examples of VPR are shown in Fig. 1. These two profiles were observed during the same event of 1-3 January 2006 and correspond to different distances from the radar site $(5$ and $18 \mathrm{~km})$ and different heights of the melting layer. The radar beamwidth is about $0.9^{\circ}$, which corresponds to about 80 - and $280-\mathrm{m}$ cross-beam resolutions at 5 and at $18 \mathrm{~km}$, respectively. As a result of these resolution differences, the $5-\mathrm{km}$ range profile (1615 UTC) has more details than the $18-\mathrm{km}$ profile (0406 UTC) and exhibits a sharper reflectivity bright band (BB), which is associated with the melting layer. The vertical beam data (not shown) indicate very similar BB thickness and strength despite differing heights (about $1.15 \mathrm{~km}$ AGL at $1615 \mathrm{UTC}$ and about $1.75 \mathrm{~km}$ AGL at 0406 UTC); therefore BB feature differences in Fig. 1 are mostly due to spatial averaging and smoothing effects that depend on range.

When radar beams used to reconstruct VPR in Fig. 1 


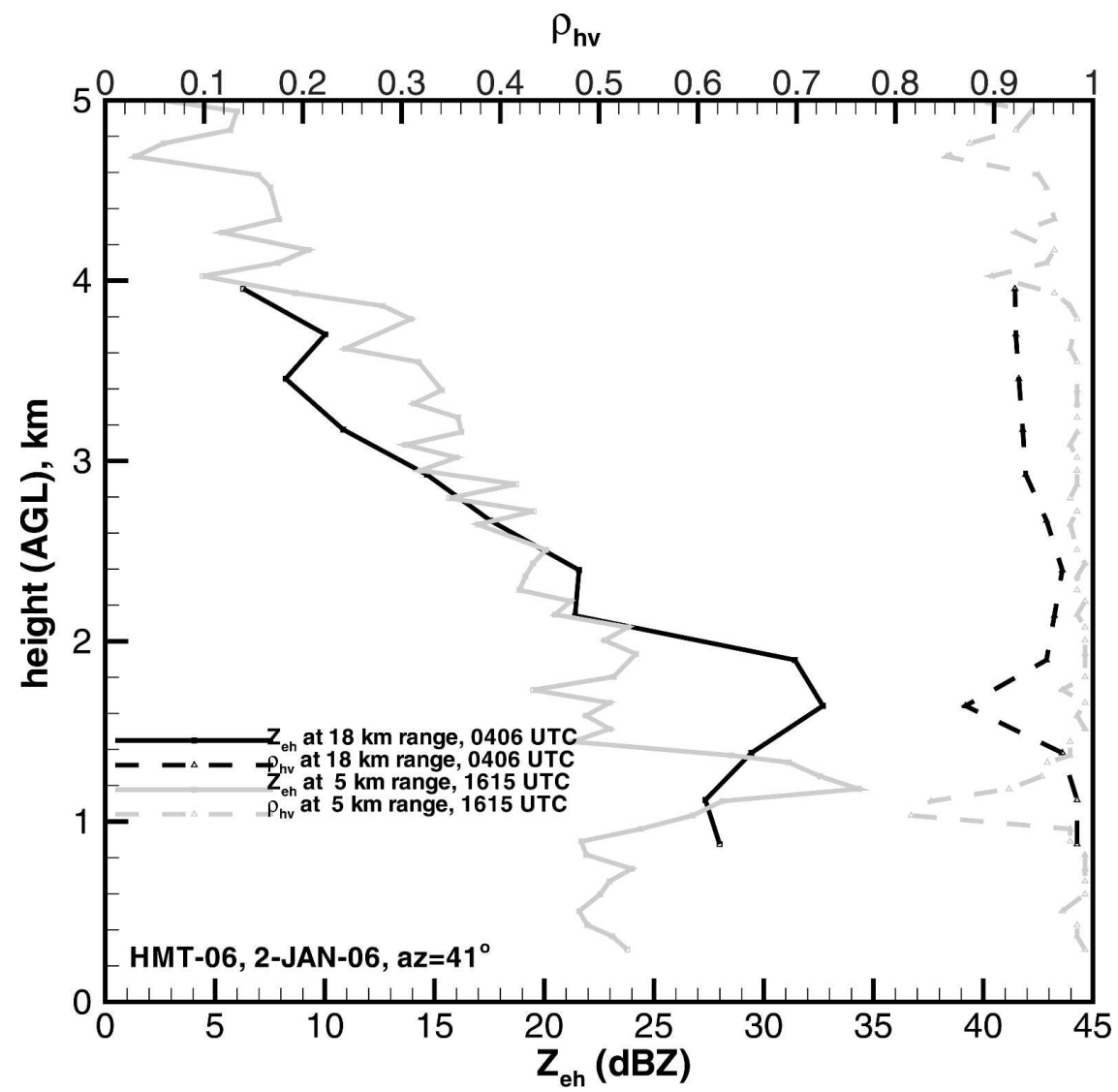

FIG. 1. Examples of individual vertical profiles of reflectivities $Z_{\text {eh }}$ and copolar correlation coefficients $\rho_{\mathrm{hv}}$ constructed from two X-band polarimetric radar RHI scans on 2 Jan 2006. Distances from the radar site and measurement times are $18 \mathrm{~km}$ and 0406 UTC (black), and $5 \mathrm{~km}$ and 1615 UTC (gray).

were penetrating rain regions, horizontal polarization reflectivities $Z_{\mathrm{eh}}$ shown in this figure were corrected for attenuation effects in rain using differential-phase-shift measurements as discussed by Matrosov et al. (2005). No correction, however, was introduced for attenuation in the melting layer and snow. While attenuation in dry snow above the melting layer is not expected to be significant because of the small imaginary parts of the complex reflective index of dry snow, mixed-phase hydrometeors in the melting layer can potentially introduce some appreciable attenuation. There currently are no efficient schemes to account for this attenuation; therefore the magnitudes of reflectivity values in $\mathrm{BB}$ and further in the snow region observed by the X-band radar might be somewhat underestimated relative to nonattenuated values. The BB attenuation effects, however, are likely not to be very strong judging from the fact that the relative BB strength $(\sim 5-7 \mathrm{~dB}$ of reflectivity increase over the rain region below) reported at weakly attenuated frequencies of $\mathrm{S}$ and $\mathrm{C}$ bands (e.g., Andrieu and Creutin 1995; Bellon et al. 2005) is com- parable to that observed with the NOAA X-band radar and shown in Fig. 1.

\section{Model mean VPR}

The RHI scans performed with the NOAA X-band radar during the HMT-06 field observations were used to construct a mean VPR at different ranges. A general form of a mean VPR is presented in Fig. 2. Note that heights in Fig. 2 are given in relation to the radar site location. Although there is some variability of reflectivity in rain below the lower boundary of the BB height $h_{0}$ in individual profiles, the average profile for $h<h_{0}$ is constant. This constant reflectivity is marked as $Z_{\text {eo }}$ in Fig. 2. This finding is in general agreement with assumptions of some other VPRs suggested in the literature (e.g., Kitchen 1997; Fabry and Zawadzki 1995).

The BB reflectivity enhancement of the mean profile is close to the triangular shape. It is assumed that the mean VPR reaches the reflectivity value $Z_{\mathrm{eo}}-\Delta Z_{1}$ at the freezing-level height $h_{1}$ (i.e., the height of the $0^{\circ} \mathrm{C}$ 


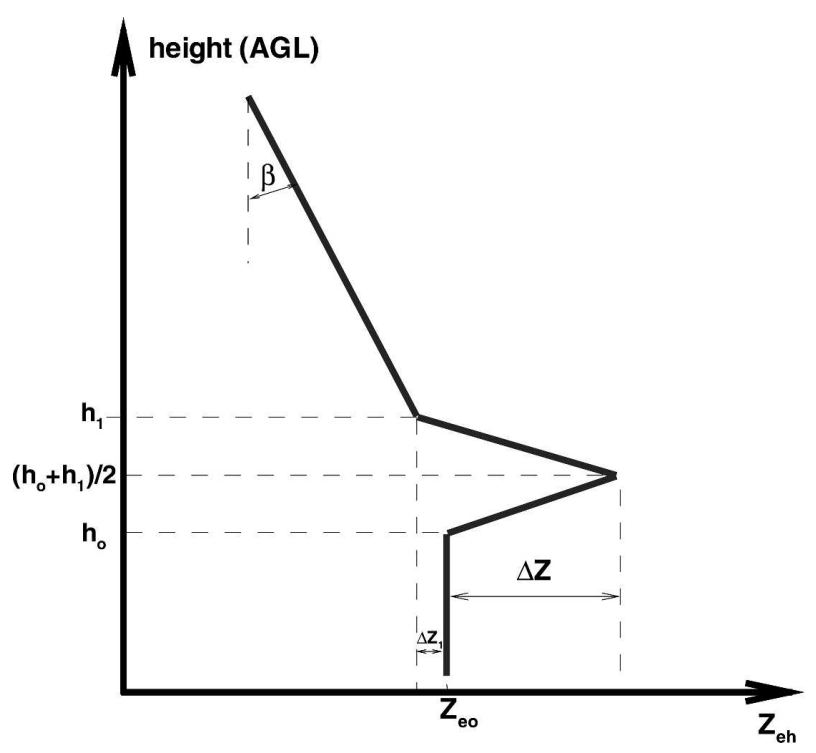

FIG. 2. An idealized mean vertical profile of reflectivity: $\Delta Z$, $Z_{\text {eo }}, \tan (\beta), h_{0}$, and $h_{1}$ are the reflectivity BB enhancement, the mean profile rain reflectivity, the mean reflectivity gradient in snow, and the lowest and highest altitudes of the melting layer, respectively.

isotherm), and then it linearly decreases in the snow region with a constant slope of $\tan (\beta)$. Fabry and Zawadzki (1995) indicate that $\Delta Z_{1}$ is on average 1-2 dB at $\mathrm{X}$ band, and so it is further used in this study that $\Delta Z_{1}=1.5 \mathrm{~dB}$. It is also assumed that the mean VPR reaches the maximum value at a height $h=\left(h_{1}+\right.$ $\left.h h_{0}\right) / 2$.

Because of smoothing effects, the maximum BB enhancement $\Delta Z$ depends on the range between the radar and the resolution volume $r$. From NOAA X-band radar RHI data the following relationship was derived:

$$
\Delta Z \approx-0.05 r+6.8,
$$

where $r$ is in kilometers and $\Delta Z$ is in decibels. The values of $r$ during HMT-06 generally varied approximately from 10 to $30 \mathrm{~km}$. The standard deviation of $\Delta Z$ values calculated using (1) is about $1.9 \mathrm{~dB}$. The value of reflectivity enhancement $\Delta Z$ can generally depend on $Z_{\text {eo }}$. Having analyzed vertical beam radar measurements, Fabry and Zawadzki (1995, their Fig. 10) indicate that $\Delta Z$ varies between 7.6 and $9.6 \mathrm{~dB}$ when $Z_{\text {eo }}$ varies between 5 and $35 \mathrm{dBZ}$, though there is no obvious trend in $\Delta Z$ as a function of $Z_{\mathrm{eo}}$. This is a modest variability in $\Delta Z$, and because no significant relation between $\Delta Z$ and $Z_{\text {eo }}$ was found based on HMT-06 data, it was further assumed that the BB enhancement does not depend on the reflectivity value in the rain region. Note that the BB reflectivity enhancement values estimated using (1) implicitly account also for the mean attenuation in the melting layer because actual (i.e., attenuated) reflectivity measurements were used in devising the relation (1).

The analysis of X-band radar RHI scans yielded a mean value of the reflectivity gradient in the snow region [i.e., $\tan (\beta)$ in Fig. 2] above the freezing level $h_{1}$ of about $5.1 \mathrm{~dB} \mathrm{~km}^{-1}$ and a standard deviation of about $2.1 \mathrm{~dB} \mathrm{~km}^{-1}$. The mean gradient value showed only a weak relation to the range $r$; therefore it was assumed to be constant and independent of $r$. The thickness of the melting layer $\Delta h$ (where $\Delta h=h_{1}-h_{0}$ ) depends on range $r$ because of a changing spatial resolution across the radar beam that degrades with $r$. In this study it is suggested, however, to determine boundaries of the melting layer $h_{1}$ and $h_{0}$ on a beam-to-beam basis using actual radar data rather than any a priori information. This suggestion reduces the amount of a priori information about parameters of VPR to a value of the BB enhancement found from (1) and the mean reflectivity gradient in the snow region $[\tan (\beta)]$.

\section{Determining melting-layer boundaries using polarimetric X-band measurements}

Because the main radar-scanning mode for the NOAA X-band radar (and any other radar) used for QPE is the low-elevation-angle sector scans, it is necessary to develop an approach for determining rain, melting-layer, and snow regions for each range gate along the radar beam. With an assumption of the mean VPR shape as in Fig. 2, this task reduces to estimations of $h_{1}$ and $h_{0}$, or the corresponding slant ranges $r_{1}$ and $r_{0}$ for the radar beam, which depend on the radar elevation angle $\alpha$ :

$$
r_{i}=h_{i} / \sin (\alpha) \text { for } i=0,1 .
$$

Note that refraction effects at the X-band ranges $(r<$ $40-50 \mathrm{~km})$ and elevation angles $\left(\alpha \geq 3^{\circ}\right)$ are negligible, and so simple geometrical considerations such as (2) can be applied in converting radar slant ranges to the height AGL relative to the radar site.

Although the mean reflectivity profile is constant below the melting layer, individual profiles may have local maxima and minima at different altitudes. Sometimes these maxima can be as pronounced as the BB itself. Positioning the $\mathrm{BB}$ along the slanted low-elevation radar beam using reflectivity data is even more challenging than for profiles because horizontal variability of rainfall (and thus the horizontal variability in corresponding $Z_{\mathrm{eh}}$ values) is often significant. This variability can significantly complicate any automatic procedure of determining the melting-layer boundaries based on reflectivity data. Although some automated BB de- 
tection procedures have been suggested for nonpolarimetric radars (e.g., Gourley and Calvert 2003), polarimetric radar measurements are more promising for efficiently discriminating among regions of rain, melting hydrometeors, and snow.

The NOAA X-band radar simultaneously transmits and simultaneously receives (STSR) the vertically and horizontally polarized radar signals. Besides differential phase, this so-called STSR measurement mode provides differential reflectivity $Z_{\mathrm{DR}}$ and the copolar correlation coefficient $\rho_{\mathrm{hv}}$. Although $Z_{\mathrm{DR}}$ usually is pronounced in the melting layer, very often layers of increased differential reflectivity, which are comparable in strength to $\mathrm{BB} Z_{\mathrm{DR}}$ values, are observed above the freezing level in the areas where single pristine crystals such as dendrites are the dominant hydrometeor habit (Matrosov 2004). This situation hampers the use of $Z_{\mathrm{DR}}$ for the purpose of unambiguous determinations of melting layers.

Brandes and Ikeda (2004) recently suggested an Sband-polarimetric-radar-based empirical procedure to detect freezing levels. In their approach they used measurements of reflectivity, linear depolarization ratio (LDR), and $\rho_{\mathrm{hv}}$ to estimate a consensus freezing-level height. The individual components of the consensus estimates based separately on reflectivity, LDR, and $\rho_{\mathrm{hv}}$ measurements show generally good agreement, though $Z_{\text {eh}}$-based estimates exhibit larger scatter (Brandes and Ikeda 2004, their Fig. 5). Because LDR measurements are not available with the NOAA X-band radar, $\rho_{\mathrm{hv}}$ is the main polarimetric parameter that can be used for efficient detection of BB and freezing-level heights and discriminating among rain, snow, and melting-level regions.

\section{a. The use of copolar correlation coefficient}

The copolar correlation coefficient $\rho_{\mathrm{hv}}$ is defined as (Bringi and Chandrasekar 2001)

$$
\rho_{\mathrm{hv}}=\left|\left\langle S_{\mathrm{hh}} S_{\mathrm{vv}}^{*}\right\rangle\right|\left(\left\langle\left|S_{\mathrm{hh}}\right|^{2}\right\rangle\left\langle\left|S_{\mathrm{vv}}\right|^{2}\right\rangle\right)^{-0.5},
$$

where $S_{\mathrm{hh}}$ and $S_{\mathrm{vv}}$ are the copolar scattering amplitudes of horizontally and vertically polarized radar signals, respectively, the angle brackets denote averaging with respect to the ensemble of hydrometeors in the radar resolution volume, and the asterisk is the complex conjugate sign.

In the STSR measurement mode, which is sometimes also called the "hybrid" mode, $\rho_{\mathrm{hv}}$ is actually measured at a zero time lag; this approach eliminates a need for assumptions that are required in the alternative transmission-simultaneous receiving mode to recalculate $\rho_{\mathrm{hv}}$ to the zero lag. Uncertainties of this coefficient result- ing from some polarization coupling present in the STSR mode are expected to be fairly small and to be negligible for the most practical observational cases (Wang et al. 2006). Note that the STSR mode was also chosen for the polarimetric upgrade of the Weather Surveillance Radar-1988 Doppler (WSR-88D) NextGeneration Weather Radar (NEXRAD) network (Doviak et al. 2000).

The magnitude of the coefficient $\rho_{\mathrm{hv}}$ is related to the variety of hydrometeor shapes present in the radar resolution volume. For an idealized situation of the ensemble of identical particles, $\rho_{\mathrm{hv}}$ is unity. Among natural hydrometeors, raindrops have the most uniform shapes, even though the degree of their oblateness depends on the drop size. The value of $\rho_{\mathrm{hv}}$ in rain is generally greater than about $0.95-0.96$, as reported in many polarimetric studies that use longer-wavelength radars such as those at $\mathrm{S}$ and $\mathrm{C}$ bands (e.g., Bringi and Chandrasekar 2001). Measurements made with the NOAA $\mathrm{X}$-band radar during all the experimental campaigns indicate similar values of $\rho_{\mathrm{hv}}$.

In addition to the reflectivity profiles, Fig. 1 also shows the corresponding profiles of $\rho_{\mathrm{hv}}$. It can be seen that in the rain region below the $\mathrm{BB}, \rho_{\mathrm{hv}}>0.95$. The magnitude of $\rho_{\mathrm{hv}}$ is generally in the range from 0.7 to 0.95 in the melting layer where a mixture of different hydrometeor shapes is present and particle sizes generally exceed those that satisfy the conditions of Rayleigh scattering. In snow, $\rho_{\mathrm{hv}}$ values are generally greater than $0.85-0.9$ with higher values expected for single-habit snowflakes such as dendrites and somewhat lower values expected for snow aggregates.

Figure 3 shows two examples of lowest-possibleelevation $\left(=3^{\circ}\right)$ slant-beam radar measurements in the azimuthal direction of $40.2^{\circ}$ where ground validation sites were located. Although measurements were taken during the same observation event of 1-3 January 2006, the melting-layer altitudes were not constant. The coldfront advection to the east was causing a gradual descent of the freezing height in the area of X-band radar measurements.

The high $\rho_{\mathrm{hv}}$ values nicely indicate the regions of rain until the range of about $23 \mathrm{~km}$ at 0243 UTC (Fig. 3a) and $16 \mathrm{~km}$ at 1627 UTC (Fig. 3b). The melting layer, which is followed by the region of snow, is confined between slant ranges of about 23 and $33 \mathrm{~km}$ at 0243 UTC and 16 and $24 \mathrm{~km}$ at 1627 UTC. When the melting layer is observed at longer radar ranges, its apparent thickness in both reflectivity and $\rho_{\mathrm{hv}}$ is larger relative to those at shorter ranges because of the smoothing and resolution effects discussed above (see Figs. 1 and 3).

The copolar correlation coefficient becomes noisier as signals approach the radar sensitivity limit (noise 

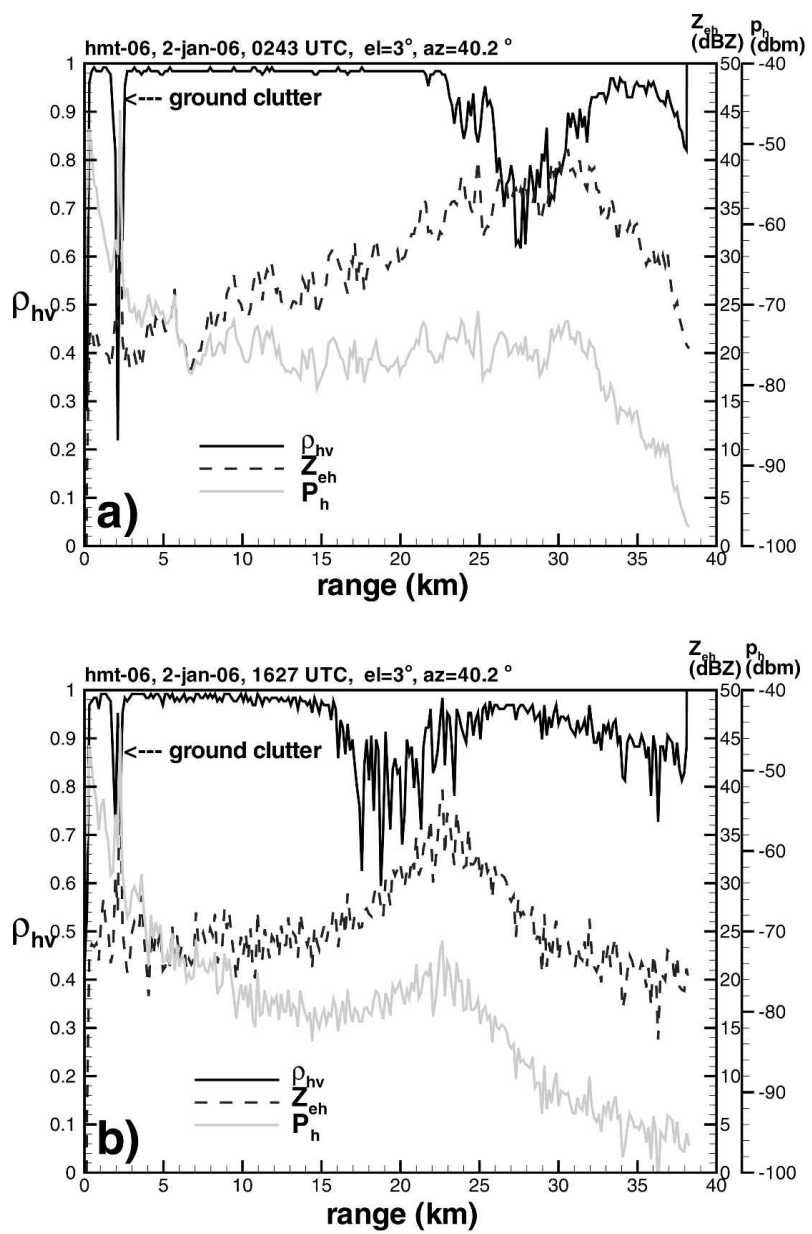

FIG. 3. Slant-beam [elevation $(\mathrm{el})=3^{\circ}$ ] range dependence of $\rho_{\mathrm{hv}}, Z_{\mathrm{eh}}$ (corrected for attenuation in rain), and the power on antenna terminals $P_{h}$ measured at (a) 0243 and (b) 1627 UTC 2 Jan 2006, with an azimuthal direction (az) of $40.2^{\circ}$.

floor), which is about $-100 \mathrm{~dB} m$ for the NOAA Xband radar (expressed in terms of the power on antenna terminals $P_{h}$ ). The spurious spikes of $\rho_{\mathrm{hv}}$ at an approximately $2-\mathrm{km}$ range is the manifestation of the ground clutter, which is characterized by low values of $\rho_{\mathrm{hv}}$ resulting from almost random phases between horizontally and vertically polarized radar echoes. The fact that these spikes represent ground clutter was also confirmed by corresponding Doppler velocity estimates, which were essentially zero, as can be expected from the stationary targets.

The $\rho_{\mathrm{hv}}$ melting-layer signature in Fig. $3 \mathrm{~b}$ is accompanied by a pronounced BB reflectivity enhancement, but such enhancement is not that obvious in Fig. 3a, where the reflectivity is only gradually increasing along the slant range until it peaks in the melting layer. This fact illustrates a generally greater robustness of using $\rho_{\mathrm{hv}}$ as the melting-layer indicator as opposed to reflec- tivity. Whereas rain values of $\rho_{\mathrm{hv}}$ are very high and stable regardless of the rain intensity, the horizontal variability of rain reflectivity and its local maxima/ minima resulting from individual rain cells can mask the $\mathrm{BB}$ features as observed along the slant radar beam. Because of the reasons given above, reflectivity is not very suitable for automated schemes of identifying rain-melting-layer-snow transitions along the slanted radar beam. It was $\rho_{\mathrm{hv}}$ that was used in this study for identifying these transitions.

As a part of this study, a relatively simple automated procedure was developed to detect melting-layer boundaries along the slant radar beam using $\rho_{\mathrm{hv}}$. In the first step, the spurious ground clutter $\rho_{\mathrm{hv}}$ returns are filtered out. The rain region is identified when $\rho_{\mathrm{hv}}$ values are steadily above 0.95 . The beginning/end ranges of the melting layer are then identified when $\rho_{\mathrm{hv}}$ stably (i.e., at least in several consecutive range gates) descends/ascends through a 0.95/0.9 threshold. As a result, slant-range boundaries of the melting layer are identified as $r_{0}^{\prime}$ and $r_{1}^{\prime}$, where the prime refers to the fact that the melting layer is identified using $\rho_{\mathrm{hv}}$.

It can be seen from Figs. 1 and $3 b$ that melting layer in reflectivity and in $\rho_{\mathrm{hv}}$ is characterized by very similar thicknesses, but they are shifted relative to each other. The $\rho_{\mathrm{hv}}$ minimum is located somewhat lower than the reflectivity maximum is. The vertical displacements of $\rho_{\mathrm{hv}}$ and reflectivity features have been commonly seen in radar polarimetric measurements (e.g., Bringi and Chandrasekar 2001). Based on X-band HMT-06, it was found that the mean value of the range displacement between the $\rho_{\mathrm{hv}}$ and $Z_{\mathrm{eh}}$ melting-layer features at the lowest beam elevation of $3^{\circ}$ is about $2 \mathrm{~km}(\Delta r=2 \mathrm{~km})$. Although there are some indications that the vertical displacement (and hence the range displacement) may weakly depend on the strength of the reflectivity maximum at S band (Brandes and Ikeda 2004), the mean value of $\Delta r$ was used in this study.

At the last step of the melting-layer identification procedure, the reflectivity $\mathrm{BB}$ range boundaries are estimated as

$$
r_{i}=r_{i}^{\prime}-\Delta r \quad \text { for } \quad i=0,1,
$$

and then (2) is used for calculating vertical height characteristics $\left(h_{0}\right.$ and $\left.h_{1}\right)$ of the mean VPR.

\section{b. An example of applying the procedure of the melting-layer/BB boundary detection}

Figure 4 shows results of the application of the $\rho_{\mathrm{hv}^{-}}$ based procedure to determine boundaries of the reflectivity $\mathrm{BB}$ for one of the experimental events during 


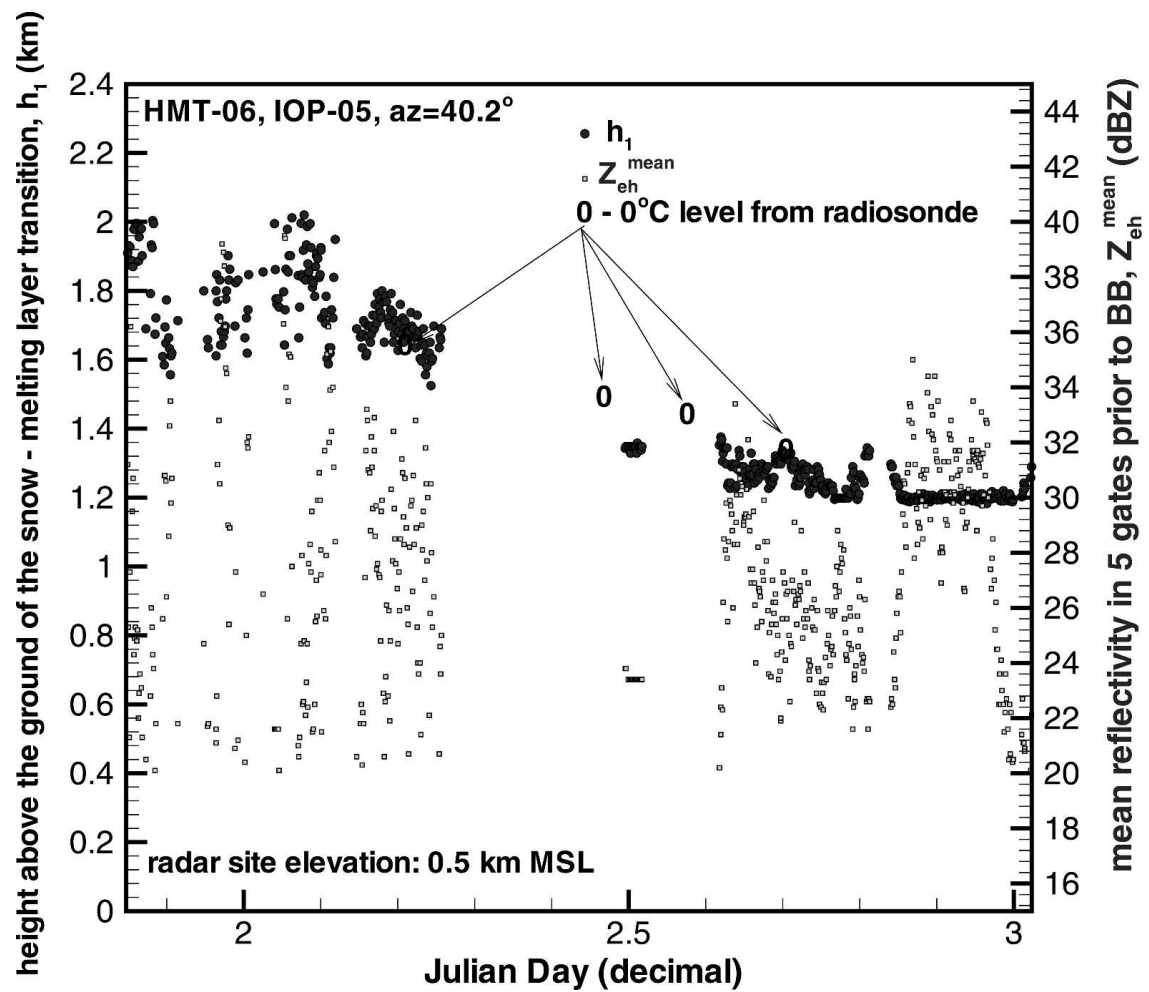

FIG. 4. Time series of the estimated heights of the BB upper boundary and the mean radar reflectivity immediately below the BB for the observational event of 1-2 Jan 2006. The $0^{\circ} \mathrm{C}$-level estimates from radiosonde soundings are superimposed.

HMT-06. This event was observed on 1-3 January 2006 and lasted for about $29 \mathrm{~h}$. Depicted is a time series of the estimated height $h_{1}$, which, as seen in Fig. 2, corresponds to the break-point transition between snow and melting-layer reflectivities and approximates the height of the freezing level. A time series of the mean reflectivity of the first five gates immediately prior to the range that corresponds to $h_{0}$ (which is where the complete transition from melting hydrometeor reflectivities to all rain reflectivities occurs) is also shown, to get a feeling of the rain intensity. This observational event consisted of two distinct rainfall periods, separated by a dry period during which no rain was observed. The upper height of the reflectivity $\mathrm{BB} h_{1}$ gradually descended from approximately 1.6-1.9 km AGL relative to the radar site in the beginning of the event to about $1.2 \mathrm{~km} \mathrm{AGL}$ at its end. During the same time, the temperature at the radar site decreased from $9.1^{\circ}$ to $5.9^{\circ} \mathrm{C}$. Figure 4 also shows freezing-level estimates from the nearby $(\sim 50 \mathrm{~km}$ away from the radar) radiosonde soundings, which also show a similar decreasing trend.

Brightband height estimates are performed for each radar beam of data. The estimates of $h_{1}$ shown in Fig. 4 correspond to the azimuthal direction of $40.2^{\circ}$. Figure 5

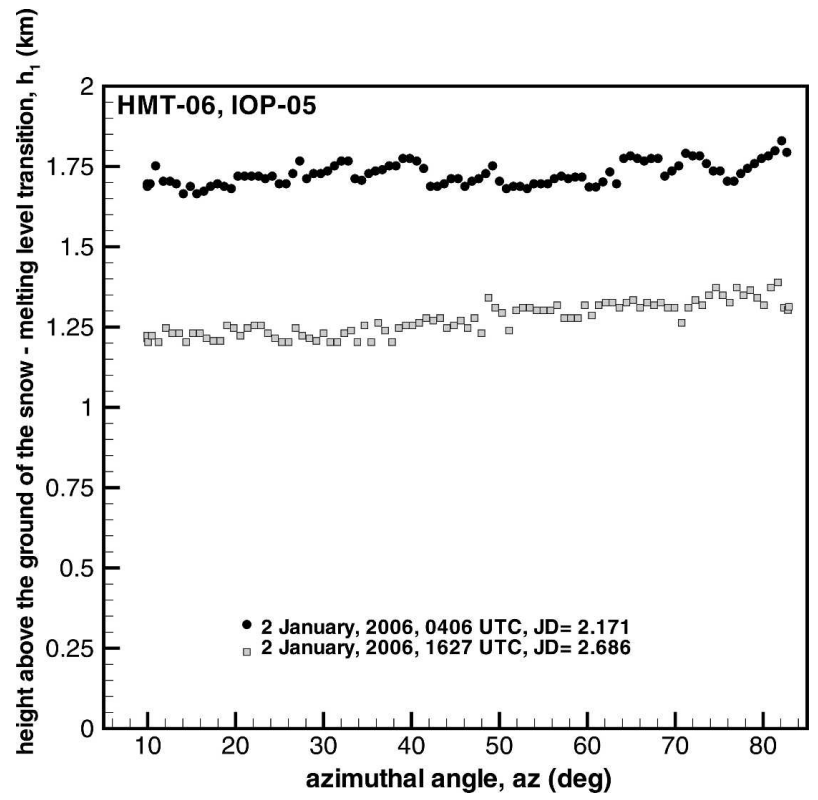

FIG. 5. The estimated heights of the reflectivity BB upper boundary relative to the radar site as a function of azimuthal angle during the HMT-06 observational event of 1-2 Jan 2006. 
depicts the reflectivity $\mathrm{BB}$ upper height estimates as a function of the azimuthal direction when the radar performed $3^{\circ}$-elevation QPE scans in the northeast quadrant $\left(0^{\circ}<\right.$ azimuth $\left.<90^{\circ}\right)$. The results are shown for representative times during both rainfall intervals. There is some weak trend of increasing $h_{1}$ as the radar beam moves from north to east, which might be caused by some orographic effects because the terrain was generally rising in the eastern direction.

On average, the variability of $h_{1}$ estimates relative to the general trend is within about $\pm 0.1 \mathrm{~km}$ (it is somewhat larger than that during the first warmer period and is smaller than that during the second colder rain period on 2-3 January 2006). The differences between $\rho_{\mathrm{hv}}$-based retrievals of $h_{1}$ and the freezing-level estimates from nearby radiosonde soundings are within
$0.1-0.2 \mathrm{~km}$. This range is comparable to S-band polarimetric estimates of freezing height levels (Brandes and Ikeda 2004).

\section{An example of applying the VPR approach}

As soon as the slant ranges of reflectivity $\mathrm{BB}\left(r_{0}\right.$ and $\left.r_{1}\right)$ and the corresponding AGL heights $\left(h_{0}\right.$ and $\left.h_{1}\right)$ are established for each radar beam using the approach discussed above, the mean VPR in (2) is applied for all of the ranges $r>r_{0}$. As a result, estimates of the rain layer reflectivity $Z_{\mathrm{eh}}^{(e)}$ (i.e., $Z_{\mathrm{eo}}$ in Fig. 2) are obtained from the actual reflectivity values $Z_{\mathrm{eh}}^{(a)}$ for all of the ranges for which the radar echoes are above the radar receiver's noise floor:

$$
\begin{aligned}
& Z_{\mathrm{eh}}^{(e)}(r)=Z_{\mathrm{eh}}^{(a)}(r) \text { for } r \leq r_{0}, \\
& Z_{\mathrm{eh}}^{(e)}(r)=Z_{\mathrm{eh}}^{(a)}(r)-2 \Delta Z\left(h-h_{0}\right)\left(h_{1}-h_{0}\right)^{-1} \text { for } r_{0}<r \leq 0.5\left(r_{0}+r_{1}\right), \\
& Z_{\mathrm{eh}}^{(e)}(r)=Z_{\mathrm{eh}}^{(a)}(r)-\Delta Z+2\left(\Delta Z+\Delta Z_{1}\right)\left(h-0.5 h_{0}-0.5 h_{1}\right)\left(h_{1}-h_{0}\right)^{-1} \text { for } 0.5\left(r_{0}+r_{1}\right)<r \leq r_{1}, \quad \text { and } \\
& Z_{\mathrm{eh}}^{(e)}(r)=Z_{\mathrm{eh}}^{(a)}(r)+\Delta Z_{1}+\tan (\beta)\left(h-h_{1}\right) \text { for } r>r_{1},
\end{aligned}
$$

where $h=r \sin (\alpha)$ and the terms $\Delta Z, \Delta Z_{1}$, and $\tan (\beta)$ were discussed in section 2 . Note that $Z_{\mathrm{eh}}^{(a)}$ values are actually observed reflectivities that were corrected for attenuation in the rain layer $\left(r<r_{0}\right)$. Equations (5b)$(5 \mathrm{~d})$ assume that it is raining at a ground location while the center of the radar beam above this location is in either the melting-layer or the snow regions.

\section{a. A note on warm rain}

Warm rain is characterized by the absence of the reflectivity BB. Often such rains are confined to a layer below the freezing level, and they have a mechanism of raindrop formation that is different from melting. Although the great majority of the experimental events during HMT-06 were characterized by stratiform rains with clearly defined melting-layer features, some examples of warm rains were also observed. In the case of warm rain along the radar beam, the values of the copolar correlation coefficient are high and are indicative of rain $\left(\rho_{\mathrm{hv}}>0.95\right)$ at all of the radar range gates where rain is observed, and no dips of $\rho_{\mathrm{hv}}$ characteristic of the melting layer are found. In such cases, the actual reflectivity values are used for QPE and no VPR correction is applied. The same is true for convective rains when upward air motions raise raindrops above the freezing level and no substantial BB features are observed.

\section{b. A $\mathrm{Z}_{\text {eh }}-\mathrm{R}$ relation for HMT-06}

The VPR approach is designed to estimate reflectivities in the underlying rain layer when the radar beam overshoots this layer and is completely or partially filled with mixed-phase hydrometeors and snow. Although it is known that the use of such polarimetric parameters as differential reflectivity $Z_{\mathrm{DR}}$ and specific differential phase shift $K_{\mathrm{DP}}$ in rain can improve QPE with both longer-wavelength radars (e.g., Ryzhkov et al. 2005) and X-band radars (e.g., Matrosov et al. 2005), there are no practical approaches to date to relate rain values of these polarimetric parameters to the corresponding values observed in melting and snow regions aloft. Because of that, this study further focuses on comparison of rainfall estimates obtained with appropriate $Z_{\mathrm{eh}}-R$ relations with and without using VPR adjustments. It is underlined, however, that, although polarimetric QPE estimators are not utilized here, the radar polarimetric measurements are used for identifying the $\mathrm{BB}$ and correcting reflectivity data for attenuation in rain.

As part of the HMT-06 field project, an impact JossWaldvogel disdrometer (JWD) was deployed at the 


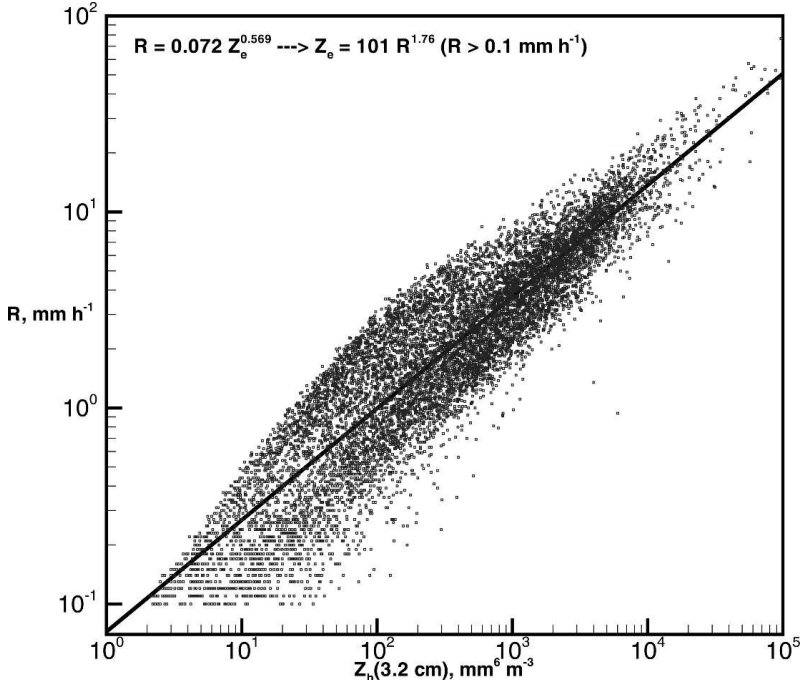

FIG. 6. The scatterplot of X-band reflectivity vs rainfall rate as calculated from the JWD DSDs collected during HMT-06 at the CFC site.

Colfax water center (CFC) site located at an elevation of $0.636 \mathrm{~km}$ MSL and at a distance of $18.3 \mathrm{~km}$ in the $40.4^{\circ}$ azimuthal direction from the radar site at Auburn (AUB). The drop size distributions (DSDs) measured by this disdrometer during HMT-06 were used to construct a mean $Z_{\mathrm{eh}}-R$ relation at $\mathrm{X}$ band. This relation $\left(Z_{\mathrm{eh}}=101 R^{1.76}\right)$ and the corresponding $Z_{\mathrm{eh}}-R$ scatterplot are shown in Fig. 6. The JWD DSDs were corrected for the effect of dead time (Sheppard and Joe 1994), and the drop shape model from Brandes et al. (2005) was used.

\section{c. Estimation of the VPR approach for the observational event of 1-3 January 2006}

Figure 7a shows the total event rainfall accumulation map calculated using the mean relation $Z_{\mathrm{eh}}=101 R^{1.76}$ without accounting for VPR for the whole 29-h period of X-band observations during the event of 1-3 January 2006. An obvious manifestation of the BB is seen as an arc of increased accumulation values centered at a radius of about $19-20 \mathrm{~km}$. Beyond the BB arc, the accumulation diminishes with range as the radar observes the snow region where reflectivity values gradually diminish with height AGL and, hence, with range. The total radar-derived accumulations at the CFC site, which is located near the center of the maximum caused by the reflectivity $\mathrm{BB}$, and at the Alta fire station site (ATA; altitude $1.09 \mathrm{~km} \mathrm{MSL}$ ) differ by more than factor of 2 in Fig. 7 a.

Figure $7 \mathrm{~b}$ shows the accumulation map obtained using the VPR approach introduced above. There are still

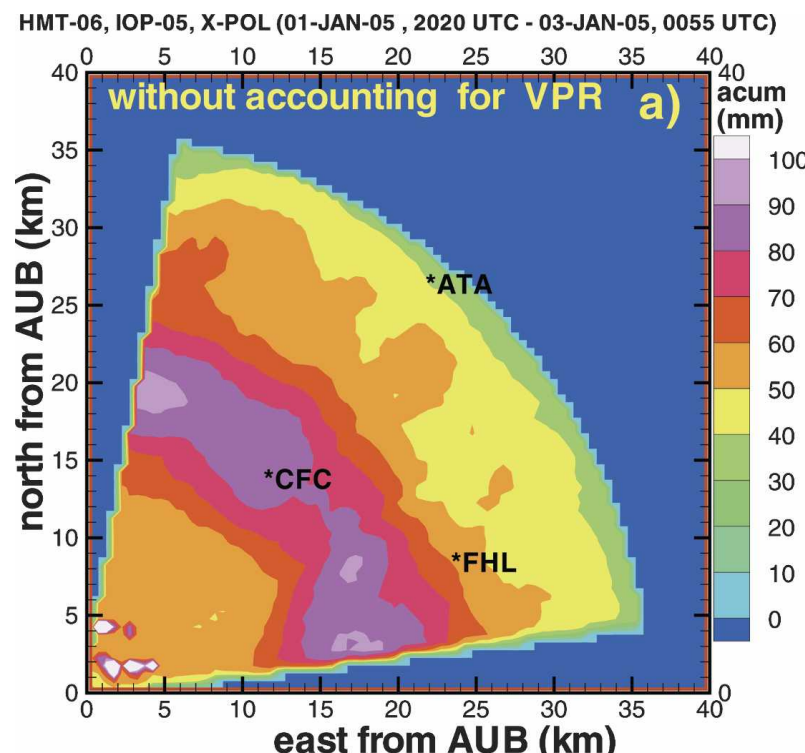

HMT-06, IOP-05, X-POL (01-JAN-05, 2020 UTC - 03-JAN-05, 0055 UTC)

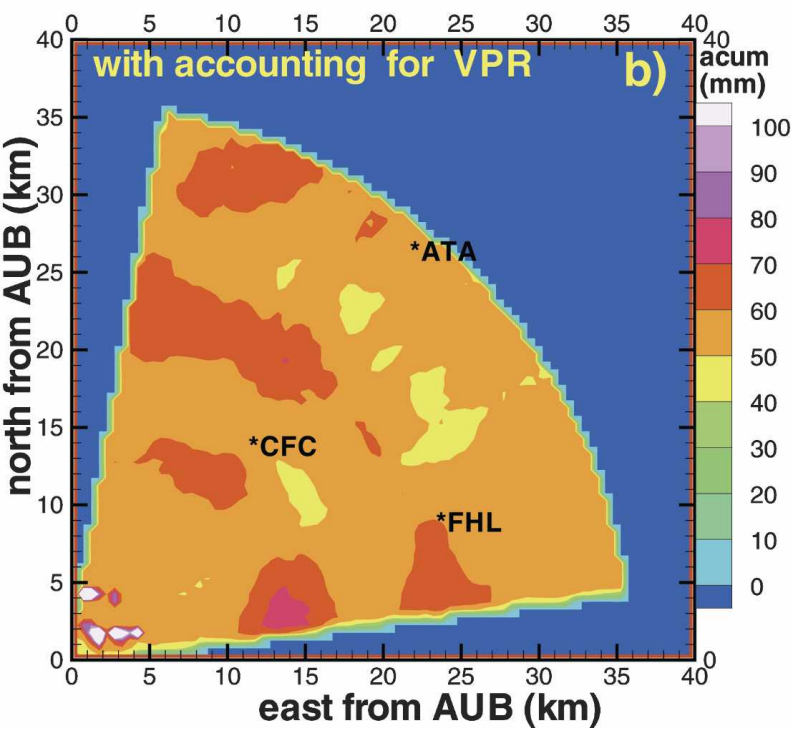

FIG. 7. The total rainfall accumulation maps for the observational event of 1-3 Jan 2006 obtained (a) without and (b) with accounting for the mean VPR.

some features that can be regarded as the manifestation of $\mathrm{BB}$, but they are much less pronounced relative to those in Fig. 7a. The total accumulation map obtained with the VPR correction is more homogeneous and does not have areas with vastly different values of accumulation. Note that the small areas of enhanced accumulation near the radar site are caused by the same ground clutter as the $\rho_{\mathrm{hv}}$ spike at $2 \mathrm{~km}$ in Fig. 3 .

Comparisons of the radar-derived total rainfall accumulations over the ground-based sites equipped with the tipping-bucket rain gauges [0.01-in. $(0.254 \mathrm{~mm})$ 
resolution] are shown in Fig. 8. In addition to the CFC and ATA sites mentioned above, comparisons are also shown for the Foresthill Divide (FHL) site located at an altitude of $0.98 \mathrm{~km}$ MSL. Ground air temperatures at all the sites throughout the event varied between $8^{\circ}$ and $2^{\circ} \mathrm{C}$, and all of the gauges were recording rain. The data for the ATA site are shown only for the second part of the event because this gauge was not operational earlier.

For the whole period shown in Fig. 8a, the radar beam over the ATA site was in the snow region above the BB. It can be seen that radar accumulation estimates obtained without the VPR correction are lower than the ground rain gauge data by more than factor of 2. The VPR correction improves the radar estimates, bringing them to a much better agreement with the ground data. During the first part of the event, when the freezing level was higher (see Fig. 4), the radar beam was generally below the $\mathrm{BB}$ at ranges corresponding to the CFC site distance, and so both radar estimates are nearly identical at this site (Fig. 8b). During the second part of the event, starting from about yearday (YD, also called "Julian day" in Figs. 4 and 8) 2.5 , the radar beam was mostly in the BB region over CFC. As a result, the rainfall accumulations obtained without the VPR correction are much higher than the ground data. Applying the VPR correction greatly improves radar estimates, bringing them much closer to the gauge data.

It can easily be seen from the freezing-level height estimates in Fig. 4, that at FHL site ranges $(\sim 25 \mathrm{~km})$, the radar beam at the $3^{\circ}$ elevation was near the lower boundary of the $\mathrm{BB}$ during the first rainy period (i.e., prior to YD 2.5), and near the upper BB boundary during the second rainy period (after YD 2.6) of the event. As can be seen from Fig. 2, the VPR corrections are smallest when the center of the radar beam is near these boundaries, which results in only small differences between the VPR-corrected and uncorrected radar estimates of rainfall accumulation (Fig. 8c).

\section{Estimation of the VPR approach performance in HTM-06}

The previous sections provide a detailed illustration of applying the VPR approach, including the BB determination with polarimetric measurements, but it is instructive to estimate an average performance of this approach on a larger dataset. During the HMT-06 field deployment from 1 December 2005 until 8 March 2006, the NOAA X-band radar measured precipitation during 13 intensive operational periods (IOPs). One of these IOPs was a snow event, and during two others the freezing level was high enough that the radar beam was
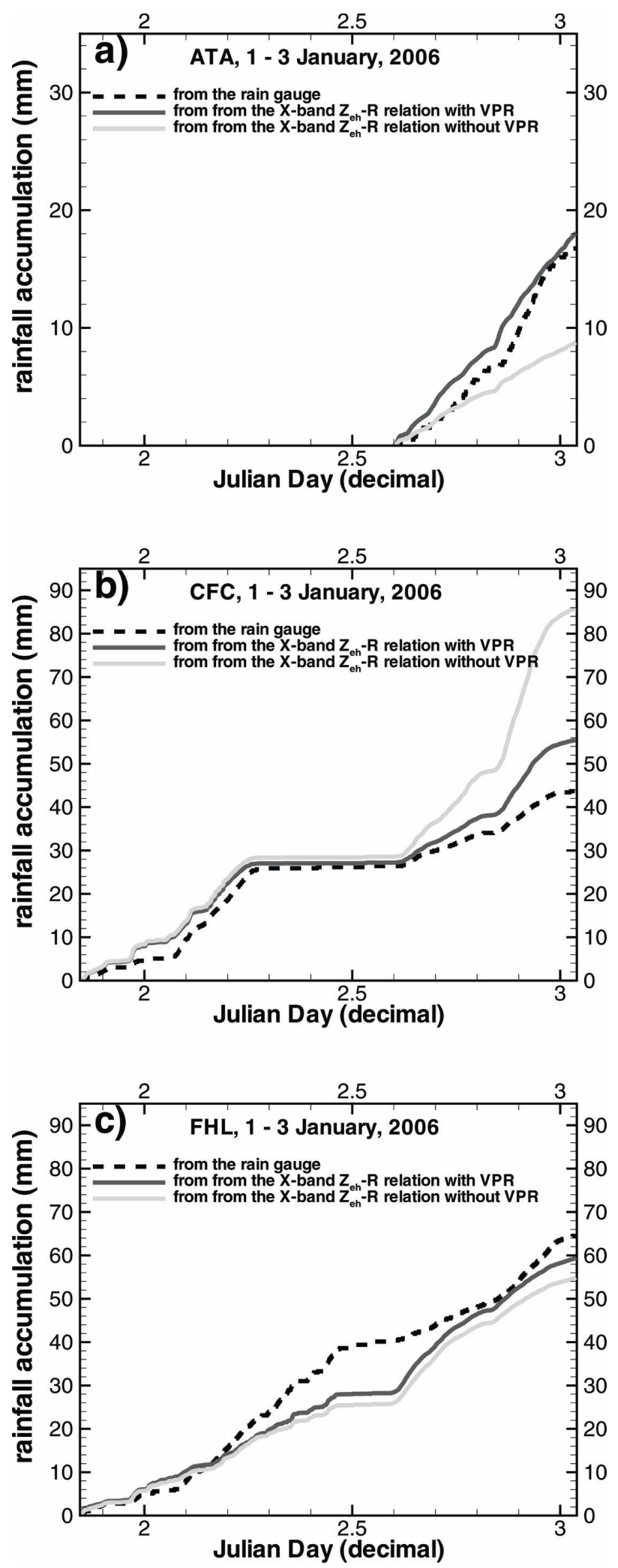

FIG. 8. Comparisons of rain gauge rainfall accumulations with the radar-derived accumulations from the mean $Z_{\mathrm{eh}}-R$ relations with and without the VPR correction for the event of 1-2 Jan 2006 at the (a) ATA, (b) CFC, and (c) FHL sites. 
TABLE 1. Total rainfall accumulations from the gauge and the radar with and without using the VPR approach with the polarimetric BB identification. The events correspond to the case in which the radar beam was sensing BB and/or snow regions while it was raining at the ground.

\begin{tabular}{|c|c|c|c|c|}
\hline $\begin{array}{c}\text { Accumulation from } Z_{\mathrm{eh}}-R \\
\text { with VPR }(\mathrm{mm})\end{array}$ & $\begin{array}{c}\text { Accumulation from } Z_{\mathrm{eh}}-R \\
\text { without VPR }(\mathrm{mm})\end{array}$ & $\begin{array}{l}\text { Accumulation from } \\
\text { gauge }(\mathrm{mm})\end{array}$ & IOP & Site \\
\hline 43.4 & 59.4 & 50.2 & 1 & ATA \\
\hline 68.2 & 64.1 & 86.9 & 2 & ATA \\
\hline 145 & 157 & 201 & 4 & ATA \\
\hline 17.4 & 8.8 & 16.8 & 5 & ATA \\
\hline 12.0 & 7.6 & 11.8 & 6 & ATA \\
\hline 36.2 & 20.4 & 47.0 & 7 & ATA \\
\hline 41.3 & 24.6 & 38.4 & 8 & ATA \\
\hline 24.3 & 31.1 & 28.9 & 10 & ATA \\
\hline 73.6 & 90.7 & 82.5 & 12 & ATA \\
\hline 73.2 & 43.6 & 73.2 & 14 & ATA \\
\hline 60.6 & 56.2 & 64.8 & 5 & FHL \\
\hline 35.3 & 28.3 & 34.8 & 7 & FHL \\
\hline 42.0 & 38.1 & 39.4 & 8 & FHL \\
\hline 48.6 & 37.2 & 52.8 & 14 & FHL \\
\hline 28.3 & 56.8 & 17.9 & 5 & CFC \\
\hline 26.9 & 48.6 & 32.5 & 7 & $\mathrm{CFC}$ \\
\hline 49.0 & 94.5 & 48.5 & 14 & CFC \\
\hline
\end{tabular}

within the rain regions at all of the ranges in the NOAA $\mathrm{X}$-band radar QPE scanning mode $(r<38 \mathrm{~km})$. For the other 10 events, the radar was sensing either BB or snow regions above the ATA site while it was raining at the ground. These events are of interest for testing the VPR approach with polarimetric BB identification. For some of the events, the freezing level was low enough that the radar beam at its lowest elevation $\left(=3^{\circ}\right)$ was above the rain regions already over the FHL and even CFC sites, which are closer to the radar. Table 1 summarizes the events that were used to estimate the performance of the approach. Note that the radar-derived information in Table 1 corresponds to the periods during which surface gauges were operational.

A relative bias (RB) and standard deviation (RSD) of the radar-derived accumulations are estimated using the following expressions:

$$
\begin{aligned}
\mathrm{RB} & =\left\langle\left(A_{R}-A_{G}\right) A_{G}^{-1}\right\rangle \times 100 \% \text { and } \\
\mathrm{RSD} & =\left\langle\left(A_{R}-A_{G}\right)^{2} A_{G}^{-2}\right\rangle^{0.5} \times 100 \%,
\end{aligned}
$$

where $A_{R}$ and $A_{G}$ are the radar- and gauge-derived accumulations, and the angle brackets denote averaging with respect to the events. The RB and RSD estimates for the mean $Z_{\mathrm{eh}}-R$ relation without VPR corrections are $4 \%$ and $65 \%$, respectively. As a rule, this relation overestimates accumulation when the radar is sensing the $\mathrm{BB}$ and underestimates it when the radar beam was in the snow region. Applying the VPR correction when $\mathrm{BB}$ is identified with the polarimetric measurements improves both estimates, resulting in $\mathrm{RB} \approx 3 \%$ and $\mathrm{RSD} \approx 32 \%$.

\section{Summary and conclusions}

The transportable polarimetric X-band radar is a useful and efficient tool for providing high-resolution rainfall parameter mapping at ranges up to $40-50 \mathrm{~km}$. Use of this radar in mountainous terrain, however, presents certain challenges because the lowest beam elevations $\left(<3^{\circ}\right)$ are often blocked and the QPE measurements need to be done at higher elevations to get the needed coverage. In combination with the lower freezing levels that are typically observed in winter rain storms in California, this effect often results in the radar beam being either in a reflectivity bright band caused by melting hydrometeors or in a snow region above the freezing level, even though it usually rains at the ground. Such situations are also common even for the lowestelevation $\left(<1^{\circ}\right)$ measurements made by the longerwavelength surveillance radars at longer ranges because of beam broadening and refraction. The a priori information about the vertical profile of reflectivity is sometimes used to recalculate the reflectivities measured aloft to their ground estimates.

In this study, the VPR approach is applied to the NOAA X-band radar measurements taken during the wintertime field project in the Sierra Nevada foothills. Unlike in many other VPR studies, the exact location of the reflectivity $\mathrm{BB}$ is estimated on a beam-to-beam basis using polarimetric measurements of $\rho_{\mathrm{hv}}$. The copolar 
correlation coefficient $\rho_{\mathrm{hv}}$ provides a robust discrimination among regions of rain, melting layer, and snow along the beam. An important advantage of copolar correlation over reflectivity for the precipitation phase separation is that $\rho_{\mathrm{hv}}$ threshold levels corresponding to phase transitions practically do not depend on precipitation intensity. Variability of precipitation along the radar beam (e.g., isolated rain cells and/or horizontal gradients of precipitation) impedes the use of reflectivity as an indicator of phase transitions.

It is shown that the $\rho_{\mathrm{hv}}$-based estimates of the reflectivity BB upper boundary, which are used as a proxy of the freezing-level height, typically agree within 100-200 $\mathrm{m}$ with $0^{\circ}$ isotherm height estimates from nearby radiosonde soundings. Because the suggested BB identification approach provides the BB boundaries for each beam, the apparent increase of the $\mathrm{BB}$ thickness with range, which is due to the cross-beam resolution issues, is automatically accounted for.

The mean X-band VPR was suggested based on the RHI scanning measurements that were periodically performed between the QPE scans. As with most climatological VPR proposed previously, this mean VPR suggests the constant reflectivity profile in the rain region below the melting layer. The mean VPR also accounts for the fact that the apparent maximum BB reflectivity enhancement $\Delta Z$ diminishes with range because of a degrading spatial resolution. Whereas the $\mathrm{X}$-band reflectivity measurements are corrected for attenuation using differential phase data in the rain region, there is no special correction in the $\mathrm{BB}$ and snow regions. Any attenuation that is present in those regions, however, is accounted for on average by the mean VPR because actual (i.e., attenuated in BB) reflectivity measurements were used in devising the VPR parameters.

The suggested VPR approach with a beam-by-beam $\mathrm{BB}$ polarimetric identification was applied to the HMT$06 \mathrm{X}$-band radar dataset using the mean $Z_{\mathrm{eh}}-R$ relation. Comparisons of the radar reflectivity-based estimates with the gauge-derived rainfall accumulations when the radar resolution volume above the gauges was either in $\mathrm{BB}$ or snow regions while it was raining at the ground indicate an approximately $32 \%$ relative standard deviation between the radar and gauge measurements. This value is about a factor-of- 2 improvement over the nonVPR-corrected estimates when the corresponding relative standard deviation was only about $65 \%$. The mean biases of both approaches are small in part because accumulation overestimations when the radar resolution volume is in the $\mathrm{BB}$ are approximately balanced by underestimations when this volume is in the snow region.
The suggested approach of separating rain, meltinglayer, and snow regions along a slanted radar beam using the copolar correlation coefficient can be applied for any polarimetric radar measuring this coefficient. The particular parameters of the mean VPR such as the $\mathrm{BB}$ reflectivity enhancement $\Delta Z$, the range dependence of this enhancement, and the snow-region reflectivity gradient $\tan (\beta)$ should be established for a particular radar and experimental setting. The results of this study can be useful for polarimetric NEXRAD applications in identifying the melting layer.

Acknowledgments. The HMT project is sponsored by NOAA. Many scientists and engineers were involved in the field operations. Special thanks are given to Brooks Martner (NOAA/ESRL) and David Jorgensen (NOAA/ NSSL) for the fieldwork coordination and for useful comments on this study.

\section{REFERENCES}

Anagnostou, E. N., M. N. Anagnostou, W. F. Krajewski, A. Kruger, and B. J. Mirovsky, 2004: High-resolution rainfall estimation from X-band polarimetric radar measurements. $J$. Hydrometeor., 5, 110-128.

Andrieu, H., and J. D. Creutin, 1995: Identification of vertical profiles of radar reflectivity for hydrological applications using an inverse method. Part I: Formulation. J. Appl. Meteor., 34, 225-239.

Bellon, A., G. W. Lee, and I. Zawadzki, 2005: Error statistics of VPR corrections in stratiform precipitation. J. Appl. Meteor., 44, 998-1015.

Brandes, E. A., and K. Ikeda, 2004: Freezing-level estimation with polarimetric radar. J. Appl. Meteor., 43, 1541-1553.

- G. Zhang, and J. Vivekanandan, 2005: Corrigendum. $J$. Appl. Meteor., 43, 1541-1553.

Bringi, V. N., and V. Chandrasekar, 2001: Polarimetric Doppler Weather Radar. Cambridge University Press, 636 pp.

Doviak, R. J., V. Bringi, A. Ryzhkov, A. Zahrai, and D. Zrnic, 2000: Considerations for polarimetric upgrades to operational WSR-88D radars. J. Atmos. Oceanic Technol., 17, 257278.

Fabry, F., and I. Zawadzki, 1995: Long-term observations of the melting layer of precipitation and their interpretation. J. Atmos. Sci., 52, 838-851.

Gourley, J. J., and C. M. Calvert, 2003: Automated detection of the bright band using WSR-88D data. Wea. Forecasting, 18, 585-598.

Kitchen, H., 1997: Towards improved radar estimates of surface precipitation at long range. Quart. J. Roy. Meteor. Soc., 123, $145-163$.

Koistinen, J., 1991: Operational correction of radar rainfall errors due to the vertical reflectivity profile. Preprints, 25th Int. Conf. on Radar Meteorology, Paris, France, Amer. Meteor. Soc., 91-94.

Matrosov, S. Y., 2004: Depolarization estimates from linear H and $\mathrm{V}$ measurements with weather radars operating in simultaneous transmission-simultaneous receiving mode. J. Atmos. Oceanic Technol., 21, 574-583.

— K. A. Clark, B. E. Martner, and A. Tokay, 2002: X-band 
polarimetric radar measurements of rainfall. J. Appl. Meteor., 41, 941-952.

—, D. E. Kingsmill, B. E. Martner, and F. M. Ralph, 2005: The utility of X-band polarimetric radar for quantitative estimates of rainfall parameters. J. Hydrometeor., 6, 248-262.

R. Cifelli, P. C. Kennedy, S. W. Nesbitt, S. A. Rutledge, V. N. Bringi, and B. E. Martner, 2006: A comparative study of rainfall retrievals based on specific differential phase shifts at X- and S-band radar frequencies. J. Atmos. Oceanic Technol., 23, 952-963.

Park, S.-G., M. Maki, K. Iwanami, V. N. Bringi, and V. Chandrasekar, 2005: Correction of radar reflectivity and differential reflectivity for rain attenuation at X band. Part II: Evalu- ation and application. J. Atmos. Oceanic Technol., 22, 16331655.

Ryzhkov, A. V., S. E. Giangrande, and T. J. Schuur, 2005: Rainfall estimation with a polarimetric prototype of WSR-88D. $J$. Appl. Meteor., 44, 502-515.

Sheppard, B. E., and P. I. Joe, 1994: Comparison of raindrop size distribution measurements by a Joss-Waldvogel disdrometer, a PMS 2DG spectrometer, and a POSS Doppler radar. $J$. Atmos. Oceanic Technol., 11, 874-887.

Wang, Y., V. Chandrasekar, and V. N. Bringi, 2006: Characterization and evaluation of hybrid polarization observation of precipitation. J. Atmos. Oceanic Technol., 23, 552-572. 